\title{
Measurement of continuous-energy neutron-incident neutron-production double-differential cross sections for Al up to several hundred MeV
}

\author{
S. Noda ${ }^{1, \text { a }}$, T. Watanabe ${ }^{1}$, N. Shigyo ${ }^{1}$, K. Ishibashi ${ }^{1}$, D. Satoh ${ }^{2}$, T. Sato ${ }^{2}$, T. Nakamura ${ }^{3}$, and R.C. Haight ${ }^{4}$ \\ 1 Kyushu University, Fukuoka, Japan \\ 2 Japan Atomic Energy Agency, Ibaraki, Japan \\ 3 Tohoku Univiersity, Sendai, Japan \\ ${ }^{4}$ Los Alamos National Laboratory, Los Alamos, NM, USA
}

\begin{abstract}
Neutron-production double-differential cross sections for aluminum were measured using continuousenergy neutron from spallation target at the WNR facility in LANSCE. Incident neutron beam was produced by $800 \mathrm{MeV}$ linac. NE213 liquid organic scintillators were utilized to detect the emitted neutron from the sample. A fission ionization chamber was set to monitor incident neutron flux. Incident neutron energy was determined by the time-of-flight method between spallation source to neutron detectors. Emitted neutron energy was obtained by the energy deposited in the neutron detector.
\end{abstract}

\section{Introduction}

As applications of intermediate energy proton, there are accelerator-driven transmutation systems (ADS), intense spallation source and cancer therapy. With intense spallation source, the further progress in material science is expected. In the intermediate energy region, secondary scattered neutron effects should be taken into account. Neutron incident data are required to estimate that effect. However, neutron incident data are scarce in this energy region. Some experimental data of $(\mathrm{n}, \mathrm{xn})$ double-differential cross sections were reported [1,2] for incident neutron energies above $20 \mathrm{MeV}$. These were performed using mono-energetic neutron beam produced by $\mathrm{T}(\mathrm{d}, \mathrm{n}){ }^{4} \mathrm{He}$ or ${ }^{7} \mathrm{Li}(\mathrm{p}, \mathrm{n})^{7} \mathrm{Be}$. Because of almost no mono-energetic neutron source and the difficulty of the neutron detection, there are not enough $(n, x n)$ data in the intermediate energy range. The objective of this study is to measure neutron-incident neutron-production $(\mathrm{n}, \mathrm{xn})$ doubledifferential cross sections (DDX) in the intermediate energy range.

\section{Experimental methods}

The experiments were carried out at the 4FP15L beam line of Weapons Neutron Research facility [3] in Los Alamos Neutron Science Center. Incident neutrons were provided from Target-4, on which spallation reaction was triggered by $800 \mathrm{MeV}$-proton beam. So the incident neutron beam energy range is up to $800 \mathrm{MeV}$. Incident neutron was transported about $90 \mathrm{~m}$ long flight path, and introduced to the experimental room through a collimator of $4 \mathrm{~cm}$ diameter. A polyethylene and a copper blocks were place on the upper beam line in order to cut-off lower energy neutrons. The sample to measure was Al. NE213 liquid organic scintillators were utilized as neutron detectors. We carried out 2 kinds of measurements

\footnotetext{
${ }^{a}$ Presenting author,

e-mail: shusaku@kune2a.nucl.kyushu-u.ac.jp
}

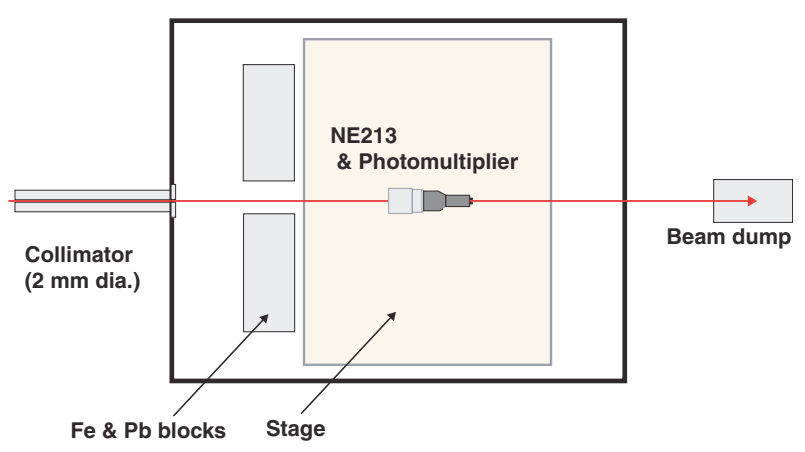

Fig. 1. Schematic view of experimental setup for response function measurement.

to get DDX, first was response function measurement, second was cross section measurement.

\subsection{Response function measurement}

The experimental setup for response function measurement is shown in figure 1 . The NE213 detectors were $12.7 \mathrm{~cm}$ thick and $12.7 \mathrm{~cm}$ diameter cylindrical, connected to Hamamatsu R1250 photomultipliers. The detectors were placed on the beam line, and the spallation neutrons were directly induced to them. The collimator of small diameter $(2 \mathrm{~mm}$ diameter $)$ was used to prevent pile-up events and damaged detectors due to extremely intense neutron incidence. In this measurement, the time-of-fight between spallation source and the detectors and the deposited charge values were obtained for all NE213 detectors.

\subsection{Cross section measurement}

The schematic view of cross section experiment is shown in figure 2 . The sample material to measure was ${ }^{\text {nat }} \mathrm{Al}(5 \mathrm{~cm}$ thick, $5 \mathrm{~cm}$ dia.). The NE213 detectors were arranged at $15^{\circ}, 30^{\circ}$, 


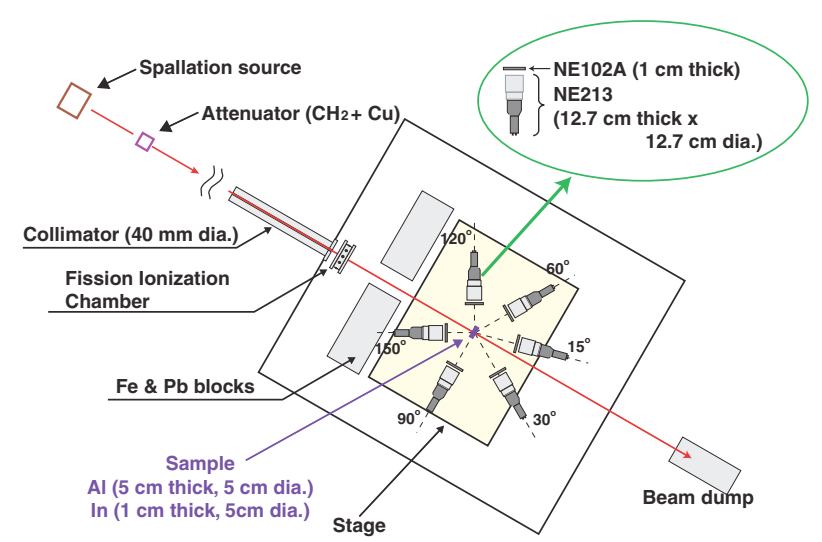

Fig. 2. Schematic view of experimental setup for cross section measurement.

$60^{\circ}, 90^{\circ}, 120^{\circ}$ and $150^{\circ}$ to detect emitted neutrons from the sample. In front of each NE213 detector, $1 \mathrm{~cm}$ thick NE102A plastic scintillators were placed as veto detectors. The distance from sample to NE213 detectors were about $0.7 \mathrm{~m}$. A fission ionization chamber [4] was set behind the collimator in order to monitor the incident neutron flux. In this measurement, we measured the time-of-flight between spallation source and the detectors and deposited charge in the NE213 detectors.

\section{Analysis}

\subsection{Fission ionization chamber}

The incident neutron flux was monitored by a fission ionization chamber. Incident-neutron dependent fission events were separated from particle-knockout or time-independent alpha-decay events in offline analysis. The neutron energy was determined by the time-of-flight. The same method as Wender's et al. [4] was applied for incident neutron flux analysis.

\subsection{NE213 liquid organic scintillator}

\subsubsection{Time-of-flight}

Typical time-of-flight spectra measured in the cross section measurement is shown in figure 3. The neutron energy was determined by the time-of-flight between spallation source and NE213 detectors. The flash gamma peak was used as a time standard.

\subsubsection{Two-gate integration method}

NE213 liquid organic scintillators are sensitive to both neutrons and gamma rays. Two-gate integration method was applied to remove the gamma ray events out of raw data. The total gate covers the whole shape of pulses from the photomultipliers, and the delayed gate covers only the tails of the pulses. The figure 4 shows that neutrons and gamma

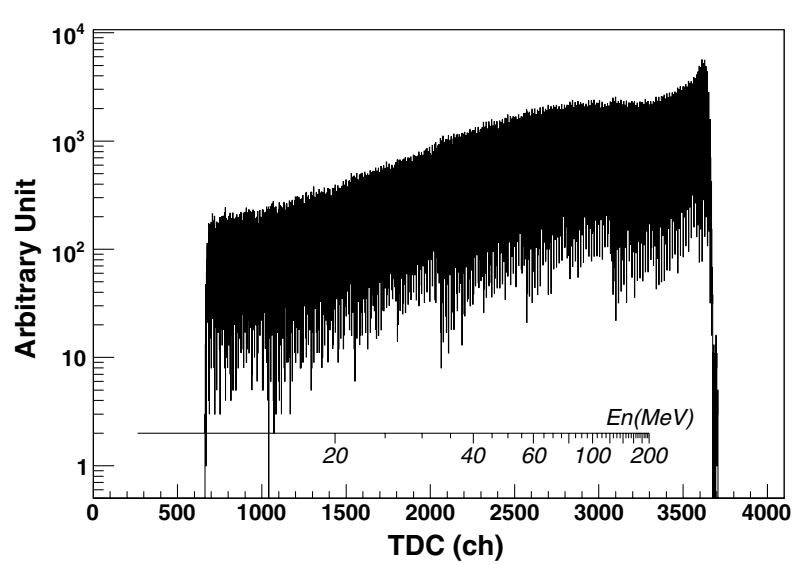

Fig. 3. Typical time-of-flight spectra.

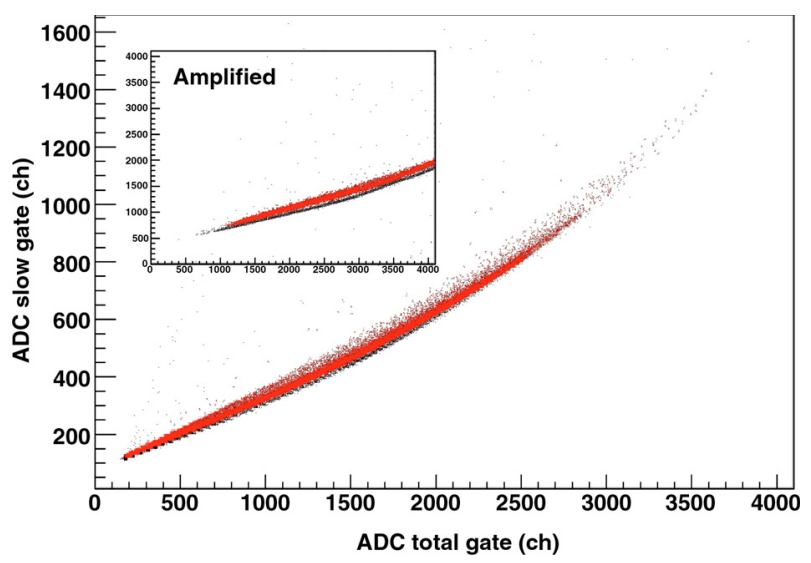

Fig. 4. Neutron and gamma ray pulse shape discrimination by the two-gate integration method. (The red dots are neutron events, the black are other particles.)

rays pulse shape discrimination by the two-gate integration method.

The deposited charge in each NE213 detector (ADC channels) were converted into the electron-equivalent light output values. In several $\mathrm{MeV}$ energy range, the Compton edges for gamma ray irradiation from ${ }^{60} \mathrm{Co}(1.25 \mathrm{MeV})$ and $\mathrm{Pu}-\mathrm{Be}(4.44 \mathrm{MeV})$ were used in calibration. At higher energy, for one incident energy the largest channel of ADC spectra corresponds to it. Response function measurement data were used to get the neutron energy corresponding to the maximum value of its ADC spectra. After getting the list of the neutron energies and ADC channels, the semiempirical formula of Cecil et al. [6] was applied to convert neutron energy into light-output $\left(\mathrm{MeV}_{e e}\right)$. The relationships between the charge information (ADC channels) and electron-equivalent light outputs is shown in figure 5.

The light output spectra for all the angles are shown in figure 6. The data were taken from the $90-110 \mathrm{MeV}$ neutron incidence on Al. Sample-out spectra were subtracted from sample-in spectra.

In response function measurement, we obtained the response function showed in figure 7. 


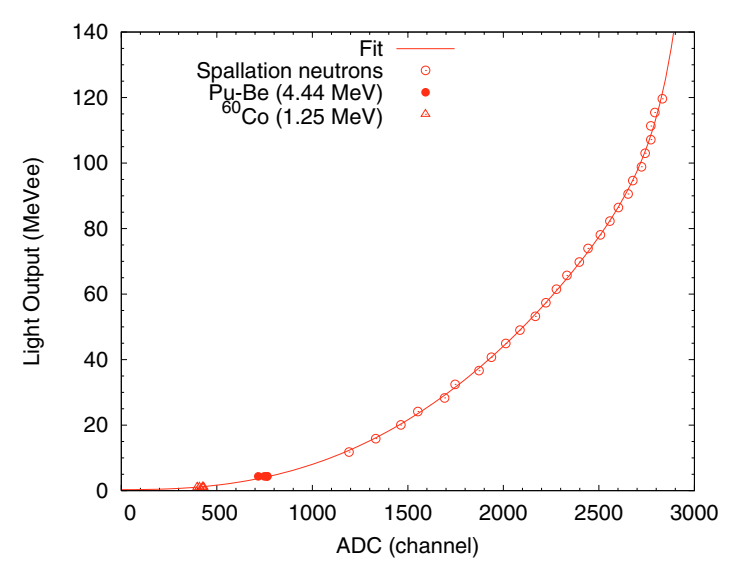

Fig. 5. Calibration curve.

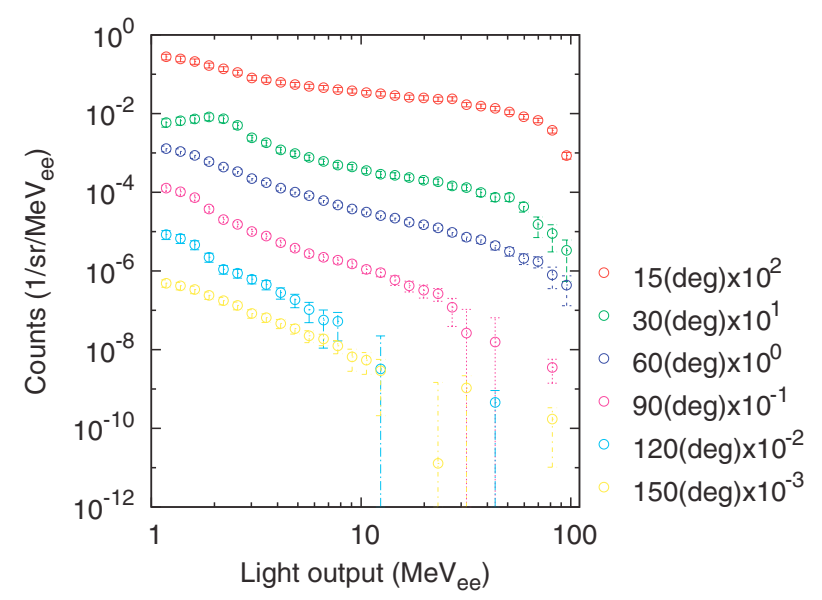

Fig. 6. Light-output spectra for $\mathrm{Al}$ at $90-110 \mathrm{MeV}$ neutron incident.

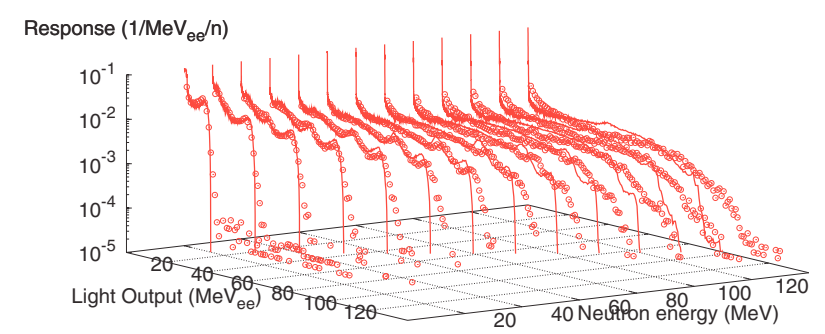

Fig. 7. Measured response functions for NE213 detector $\left(15^{\circ}\right)$ with SCINFUL-QMD calculation results.

\subsection{Unfolding}

When unfolding the light-output spectrum with the measured response functions, we assumed the Moving Source model [7] for DDX. The Moving Source model is shown below,

$\left(\frac{d^{2} \sigma}{d \Omega d E_{\text {kin. }}}\right)_{\mathrm{MS}}=\sum_{i=1}^{3} p A_{1} \exp \left\{-\left(\frac{E_{\text {kin. }}+m-p \beta_{i} \cos \theta}{\left(1-\beta^{2}\right)^{\frac{1}{2}}}-m\right) / T_{i}\right\}$.

$i=1$ stands for cascade process, $i=2$ for pre-equilibrium and $i=3$ for evaporation. $A_{i}$ is amplitude parameter, $T_{i}$ is temperature parameter, $\beta_{i}$ is velocity parameter. With the Moving Source model, we assumed the outgoing neutron distribution. Elastic scattering effects were estimated from the

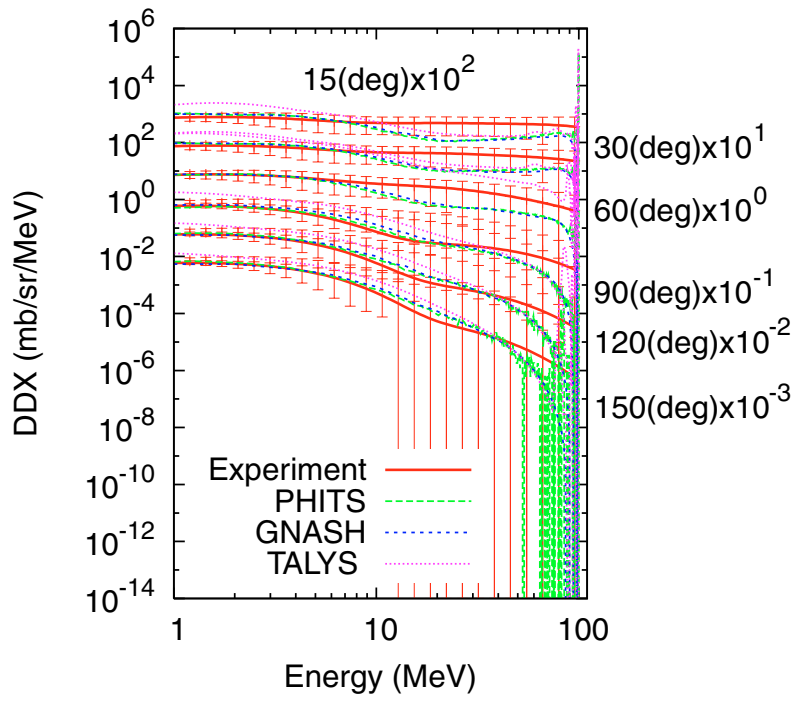

Fig. 8. Measured double-differential cross sections at incident energy 90-110 MeV on Al. They are compared with the PHITS, the GNASH and the TALYS results.

PHITS results prior to this unfolding process. In figure 8, DDX for $\mathrm{Al}$ is shown.

\section{Results and discussion}

The neutron-incident neutron-production double-differential cross sections are shown in figure 8 for incident energy of 90 $110 \mathrm{MeV}$ on $\mathrm{Al}$ sample. The experimental data were compared with the PHITS, the GNASH and the TALYS calculation results. The experimental results produce reasonable agreements with the calculation results at low energy range.

\section{Summary}

We measured the neutron-incident neutron-production doubledifferential cross sections for $\mathrm{Al}$ sample in the intermediate energy range. The spallation neutron source was employed as incident particles. NE213 liquid organic scintillator was utilized as neutron detector. Incident neutron energy was derived by the time-of-flight method. Emitted neutron energy was determined by the deposition energy in NE213 detectors. The response functions were also measured for all the NE213 detectors. Assuming the Moving Source model, with measured response functions we unfolded the light-output spectra to get DDX. Elastic scattering effects were estimated by the PHITS results. The experimental results were compared with the PHITS, the GNASH and the TALYS calculation results. In low energy range, they are in good agreement.

This work has benefited from the use of the Los Alamos Neutron Science Center at the Los Alamos National Laboratory. This facility is funded by the US Department of Energy. 


\section{References}

1. A. Marcinkowski et al., Nucl. Sci. Eng. 83, 13 (1983).

2. E.L. Hjort et al., Phys. Rev. C 53, 237 (1996).

3. P.W. Lisowski et al., Nucl. Sci. Eng. 106, 208 (1990).
4. S.A. Wender et al., Nucl. Instrum. Meth. Phys. Res. A 336, 226 (1994).

5. M. Moszynski et al., Nucl. Instrum. Meth. A 343, 563 (1994).

6. R.A. Cecil et al., Nucl. Instrum. Meth. 161, 439 (1979).

7. I.G. Bogatskaya et al., Phys. Rev. C 22, 209 (1980). 